\title{
A Method for Monitoring the Underground Mining Position Based on the Blasting Source Location
}

\author{
Xiu-zhi Meng ${ }^{1,2}$, Zong-sheng Wang ${ }^{2}$, Zeng-zhi Zhang ${ }^{1}$, Feng-qian Wang \\ ${ }^{1}$ School of Mechanical Electronic and Information Engineering, China University of Mining \& Technology, \\ Xueyuan Road, NO.11, 100083, Beijing, China, 168mxz@163.com, z.zengzhi@163.com \\ ${ }^{2}$ College of Mechanical and Electronic Engineering, Shandong University of Science and technology, \\ Qianwangang Road, NO.579, 266590, Qingdao, China, seeiteasy01@163.com
}

\begin{abstract}
Some small and medium-sized coal mines are mining beyond their mining boundary driven by profit. The illegal activities cause many mine disasters but effective supervision is very hard to achieve, especially for underground coal mining. Nowadays, artificial blasting operation is widely used in tunneling or mining in small and medium-sized coal mines. A method for monitoring the underground mining position by monitoring the blasting source position is firstly introduced in this paper. The blasting vibration waves are picked up by the detectors and dealt by the signal acquisition sub-station, and then sent to the principal computer. The blasting source is located by the principal computer and displayed in the mine's electronic map. The blasting source position is located in 10 seconds after the first $P$ wave reaching the detector, whose error is registered within 20 meters by field-proven method. Auto-monitoring of the underground mining position in real-time is solved better and management level is improved using this method.
\end{abstract}

Keywords: Blasting vibration wave, real-time monitoring, underground mining positioning, blasting source location

\section{INTRODUCTION}

$\mathrm{T}$ HE NUMBER of small and medium-sized coal mines is nearly 20,000 because of the state policy and mining technical background in the 1980's. In recent years, the state has taken measures to shut down some coal mines by rectification and integration of coal resources to improve the mining efficiency and reduce safety accidents. But until 2010, the number of small and medium-sized coal mines was still nearly 10,000 in the country and most of them were underground coal mines which were concealed and it was very difficult to supervise them effectively. In this case, many coal mines are mining beyond their mining boundary driven by profit. The illegal activities not only lead to a waste of resources and environmental damage, but also cause many safety accidents [1]. According to incomplete statistics on the webpages, 13 major safety accidents with 280 deaths were caused by ultra-layer \& cross-border mining since 2004, which resulted in an extremely bad influence.

Until now, regulatory approaches for the order of coal mining are mainly on-site supervision, such as on-the-spot underground inspection, or the detection of mineral production, etc. Because the number of coal mines is much larger than the number of regulatory staff, fully effective supervision cannot be achieved. Therefore, the use of new monitoring techniques to improve monitoring capacity is imminent.

The small and medium-sized coal mines still use blasting operations to mine due to their mining environment and economic strength. Under these circumstances, a method for locating underground mining by monitoring the blasting source position is firstly discussed in this paper. The vibration source location is the research hotspot in micro seismic monitoring technology that emerged in the last two decades and is used in development of oil field, or disaster prevention in underground mines, [2-4] etc. Monitoring underground mining position based on the micro seismic monitoring technology has not been reported in literature. The method is feasible, for no one gets involved and automonitoring the underground mining position is done in realtime by field-proven method.

\section{METHOD}

The working principle and diagram for locating the blasting source is shown in Fig.1. The five acceleration vibration sensors as detection devices buried underground orderly in the mining area pick up blasting vibration waves coming from blasting operation, and each detector is connected to a signal acquisition sub-station which uses wavelet transform to denoise the detector output signals and adopts the energy ratio method to determine the arrival time of $\mathrm{P}$ waves to the apropriate detector. In order to reduce location error, GPS time service module calibrates the clock of sub-stations and principal computer. The principal computer regulates each sub-station working status, inquires the data of the arrival time of the $\mathrm{P}$ wave, locates the bursting point by the linear positioning algorithm and translates spatial and temporal parameters of the blasting point through RS232 to the server. The server displays the bursting point's spatial message in the mine's electronic map. In addition, early warning area of the super-layer \& cross-border is drawn in the mining area boundary. When the blasting position is detected in the warning area, the principal computer sends warning information via text message to the supervising officer on the phone through the GPRS module by means of GPRS mobile communication base station, and issues a corresponding sound and light alarm signal. 


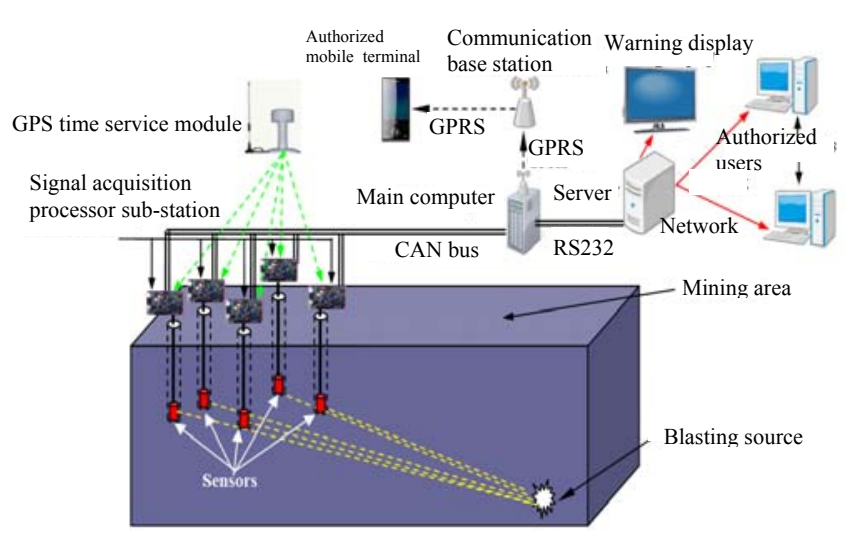

Fig.1. The working principle and diagram for locating blasting source

The blasting source location is the core content in the whole monitoring scheme. The detailed description source location is given as follows.

\subsection{Signal collection and identification}

The collection and identification of the blasting seismic wave is a key feature in the monitoring method. The detector picks up not only blasting vibration signals but also interference signals such as external interference vibration wave signals. To identify the blasting seismic wave, the interference signals must be filtered. Wavelet transform has the characteristics of low entropy, multiresolution, decorrelation and flexibility of selecting wavelet basis, but also has the analysis capabilities of the local high-resolution in the time-frequency domain, so the wavelet analysis is a good tool to analyze non-stationary and non-linear signal in the time-frequency domain [5-10]. The blasting vibration signal belongs to non-stationary signal because of short duration, fast mutation and so on. The paper takes advantage of the wavelet threshold denoising to deal with the detector output signals. The denoising principle is as follows. First, a threshold $\lambda$ is set. If the wavelet transform coefficient is greater than $\lambda$, this coefficient is mainly caused by the blasting vibration signal and kept. Otherwise, the coefficient is mainly caused by noise and is set to zero. Then, the signal is reconstructed by reserved wavelet coefficients to retain the blasting vibration signal after denoising. The denoising process is shown in Fig.2.

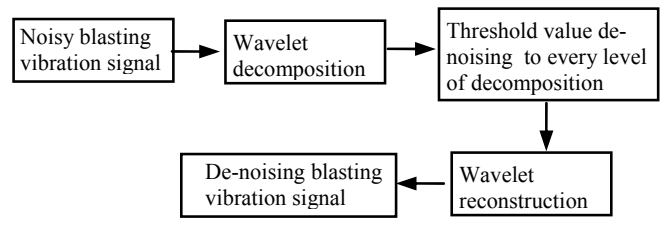

Fig.2. The denoising process

The wavelet threshold denoising methods are divided into soft threshold denoising and hard threshold denoising. The expression is as follows:
The soft threshold function:

$$
\hat{w}_{j, k}=\left\{\begin{array}{cl}
\operatorname{sign}\left(w_{j, k}\right)\left(\left|w_{j, k}\right|-\lambda\right) & \left|w_{j, k}\right| \geq \lambda \\
0 & \left|w_{j, k}\right|<\lambda
\end{array}\right.
$$

The hard threshold function:

$$
\hat{w}_{j, k}=\left\{\begin{array}{cc}
w_{j, k} & \left|w_{j, k}\right| \geq \lambda \\
0 & \left|w_{j, k}\right|<\lambda
\end{array}\right.
$$

$w_{j, k}$ are wavelet decomposition coefficients, and $\hat{w}_{j, k}$ are treatment wavelet decomposition coefficients.

Threshold value $\lambda$ is dealt by the following expression:

$$
\lambda=\sigma \sqrt{2 \ln N}
$$

Where $\sigma$ is blasting vibration signal variance, $N$ is decomposition scale.

The two methods have advantages and disadvantages: The treatment wavelet coefficient has discontinuity because of hard threshold nature. The denoising signal still contains obvious noise signal, but it can retain the details of local information. The treatment wavelet coefficient dealt by soft threshold has better continuity than that by hard threshold, but its derivative is not continuous. There is constant deviation between the treatment wavelet coefficient and the wavelet coefficient with noise, and the coefficient greater than the threshold does not comply with the fact that noise is reduced with the increase of wavelet coefficient [11-12]. The details of the blasting vibration signal must be kept to identify the time of the $\mathrm{P}$ wave reaching the detector; after comprehensive comparison, the hard threshold denoising method is used in this paper.

\subsection{Determination of arrival time}

The arrival time of the $\mathrm{P}$ wave to the detector is the detector's temporal parameter for blasting source inversion, so the determination of arrival time is a critical factor for locating the blasting source. The blasting vibration signal has high signal-to-noise ratio through the earth's low-pass filtering, the circuit anti-aliasing filtering and wavelet threshold denoising. The energy ratio is an effective and commonly used method to determine the arrival time of the $\mathrm{P}$ wave for high signal-to-noise ratio signal. The ratio of short time window average value (STA) to long time window average value (LTA) is calculated in the adjustable length moving time window for absolute value of the reconstructed blasting vibration signal. The short time window average value changing is quicker than the long time window average value changing at the $\mathrm{P}$ wave arrival time. When the ratio is larger than the designed threshold value, the arrival of $\mathrm{P}$ wave can be considered and the time is recorded. 
Suppose the reconstructed blasting vibration signal sequence is $X n$, the expressions of the STA sequence and LTA sequence are as follows:

$$
\begin{aligned}
& \operatorname{STA}(n)=\left(\sum_{i=n-m}^{n}\left|X_{i}\right|\right) / m \\
& \operatorname{LTA}(n)=\left(\sum_{i=n-l}^{n}\left|X_{i}\right|\right) / l
\end{aligned}
$$

Where $m$ means the acquisition points in the short time window, $l$ means the acquisition points in the long time window and $\left|X_{i}\right|$ is the value of the characteristic function at the moment $i$. The length of STA is expressed by $m \times d t$ and the length of LTA is expressed by $l \times d t$ (where $d t$ is the sampling time interval). The value of length determined must be combined with the characteristics of the characteristic function and calculation method of STA and LTA.

\subsection{Location of blasting point}

Every detector's spatial parameters $\left(x_{i}, y_{i}, z_{i}\right)$ are measured when the sensor is buried underground and the temporal parameter $t_{i}$ is recorded by the signal acquisition sub-station after the blasting occurs (the subscript $i$ means the $i$-th detector). Supposed the $\mathrm{P}$ wave average spread speed is $v_{p}$ and the blasting source's spatial and temporal parameters are $(x, y, z, t)$, based on linear position algorithm, the distance equation between the source and the $i$-th detector is:

$$
\left[\left(x_{i}-x\right)^{2}+\left(y_{i}-y\right)^{2}+\left(z_{i}-z\right)^{2}\right]^{\frac{1}{2}}=v_{p}\left(t_{i}-t\right)(\mathrm{i}=1,2, \ldots, 5)
$$

The (6) is a nonlinear equation. It is more difficult to handle the nonlinear problems using the general method. In addition, the spatial and temporal parameters of each detector affect positioning accuracy of the blasting source. So the paper uses the square of distance between the blasting source and the $i$-th detector minus the square of distance between the source and the $(i+1)$-th detector, which can offset the parameter error of each sensor, then (6) translates into linear equations. Matrix representation is:

$$
A X=B
$$

There is:

$$
\begin{gathered}
A=\left[\begin{array}{llll}
2\left(x_{2}-x_{1}\right) & 2\left(y_{2}-y_{1}\right) & 2\left(z_{2}-z_{1}\right) & 2 v_{P}{ }^{2}\left(t_{2}-t_{1}\right) \\
2\left(x_{3}-x_{2}\right) & 2\left(y_{3}-y_{2}\right) & 2\left(z_{3}-z_{2}\right) & 2 v_{P}{ }^{2}\left(t_{3}-t_{2}\right) \\
2\left(x_{4}-x_{3}\right) & 2\left(y_{4}-y_{3}\right) & 2\left(z_{4}-z_{3}\right) & 2 v_{P}{ }^{2}\left(t_{4}-t_{3}\right) \\
2\left(x_{5}-x_{4}\right) & 2\left(y_{5}-y_{4}\right) & 2\left(z_{5}-z_{4}\right) & 2 v_{P}{ }^{2}\left(t_{5}-t_{4}\right)
\end{array}\right] \\
X=\left[\begin{array}{llll}
x & y & z & t
\end{array}\right]^{T}
\end{gathered}
$$

$$
B=\left[\begin{array}{c}
\left(x_{2}{ }^{2}-x_{1}{ }^{2}\right)+\left(y_{2}{ }^{2}-y_{1}{ }^{2}\right)+\left(z_{2}{ }^{2}-z_{1}{ }^{2}\right)-v_{p}{ }^{2}\left(t_{2}{ }^{2}-t_{1}{ }^{2}\right) \\
\left(x_{3}{ }^{2}-x_{2}{ }^{2}\right)+\left(y_{3}{ }^{2}-y_{2}{ }^{2}\right)+\left(z_{3}{ }^{2}-z_{2}{ }^{2}\right)-v_{p}{ }^{2}\left(t_{3}{ }^{2}-t_{2}{ }^{2}\right) \\
\left(x_{4}{ }^{2}-x_{3}{ }^{2}\right)+\left(y_{4}{ }^{2}-y_{3}{ }^{2}\right)+\left(z_{4}{ }^{2}-z_{3}{ }^{2}\right)-v_{p}{ }^{2}\left(t_{4}{ }^{2}-t_{3}{ }^{2}\right) \\
\left(x_{5}{ }^{2}-x_{4}{ }^{2}\right)+\left(y_{5}{ }^{2}-y_{4}{ }^{2}\right)+\left(z_{5}{ }^{2}-z_{4}{ }^{2}\right)-v_{p}{ }^{2}\left(t_{5}{ }^{2}-t_{4}{ }^{2}\right)
\end{array}\right]
$$

In general, the above matrix is an ill-conditioned matrix. Many methods are involved in solving this kind of equations, such as direct methods, iterative method, conjugate vector method, or some advanced algorithms, namely, genetic algorithms. Each algorithm has its own limitation. Conjugate vector method is employed to solve this matrix [13].

\section{MONITORING SYSTEM}

The monitoring system includes the following sections: vibration sensors, signal acquisition processor sub-station, principal computer, communication circuit between substation and principal computer, and power supply module and so on. The system structure diagram is shown in Fig.3. The physical map is shown in Fig.4. and the system function is shown in Fig.5.

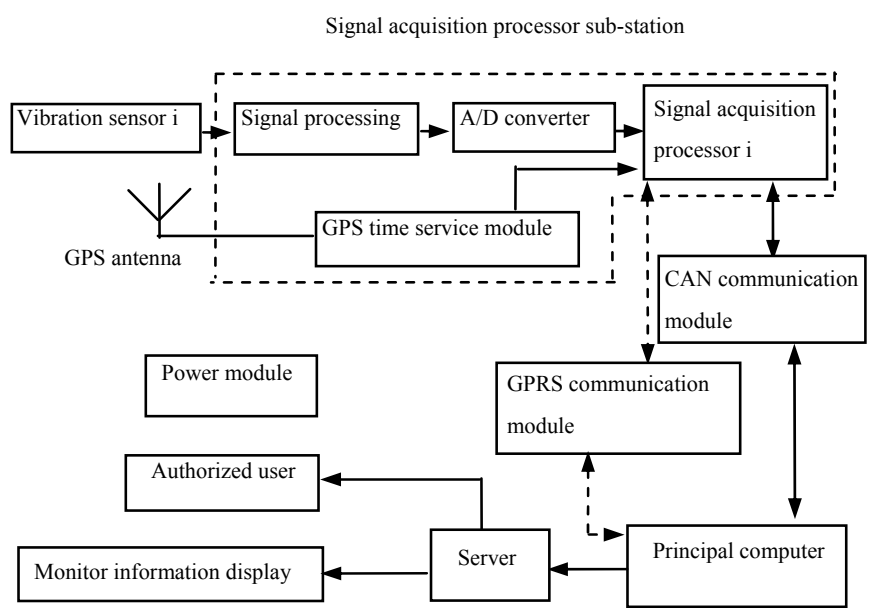

Fig.3. Diagram of monitoring system structure

The application software is designed to get the explosion position, to decide whether an explosion is beyond the border, and to show the explosion on the mine zone map. The detail is as follows:

(1) To show the explosion position. The mine zones and the distributions are printed on a big screen, which can be zoomed in and zoomed out in vectors.

(2) To alarm when a cross-border occurs. When a crossborder occurs, there is alarm and a red signal will be shown on the computer screen.

(3) To record the explosion positions and the explosion times, and to look up the records. All of the explosion positions are automatically recorded by the system, which can be looked up at any time. The system can also print the exposition tracks when needed.

(4) To warn supervising officer by text messages. When there are cross-borders, the system automatically sends messages to the supervisors by mobile phones. 


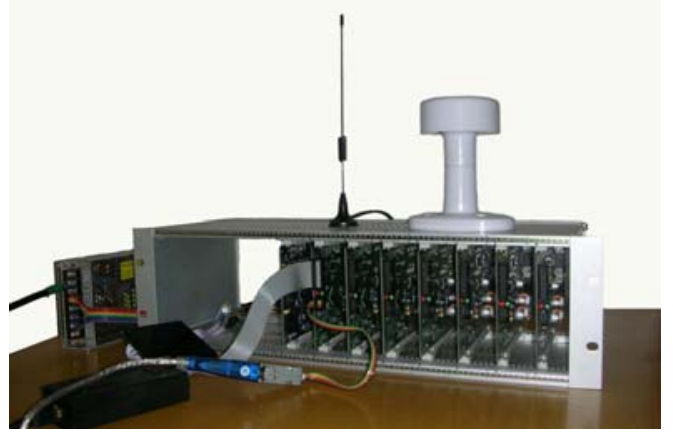

Fig.4. The System physical map

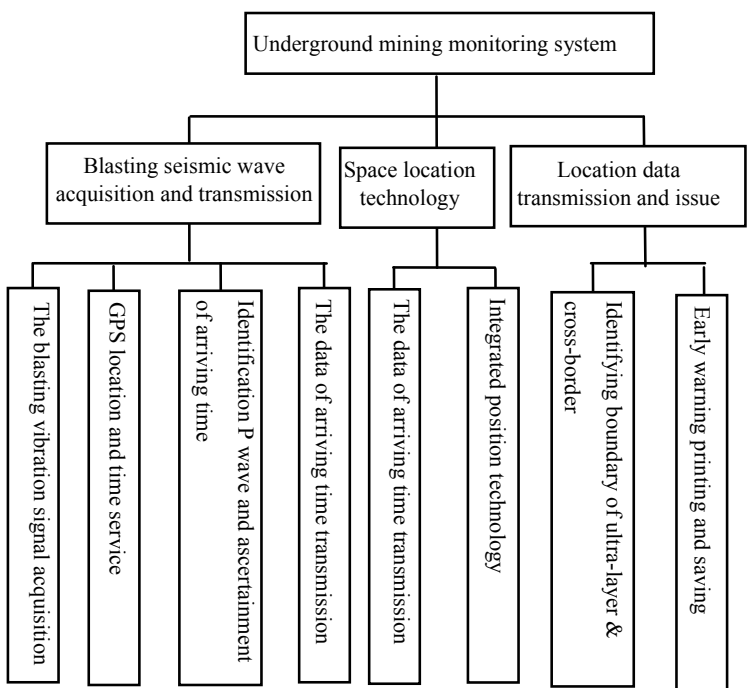

Fig.5. System function diagram

\section{INSTANCES OF APPLICATIONS}

The whole system has been tested in No. 1 mine Xin Yue Corp, Zhangqiu, China, by back to back test method. The five accelerometers are buried in five vertical wells with different depths. To decrease the location errors, the 3dimension coordinates of the five accelerometers must be as accurate as possible. Table 1. shows the exact coordinates of the five accelerometers.

Table 1 . The exactly coordinates of the five accelerometers

\begin{tabular}{|c|c|c|c|c|c|}
\hline \multirow{2}{*}{$\begin{array}{c}\text { Station } \\
\text { name }\end{array}$} & \multicolumn{3}{|c|}{ Coordinate (1954 Beijing) } & \multirow{2}{*}{$\begin{array}{l}\text { Actual depth } \\
\text { of sensor from } \\
\text { ground } \\
\end{array}$} & \multirow{2}{*}{$\begin{array}{c}\text { Actual } \\
\text { elevation of } \\
\text { sensors }\end{array}$} \\
\hline & $\begin{array}{c}X \\
\text { (north) }\end{array}$ & $\begin{array}{c}Y \\
\text { (east) }\end{array}$ & $\begin{array}{c}Z \\
\text { (elevation) }\end{array}$ & & \\
\hline $\mathrm{A} 1$ & 4061981.5 & 20556377.3 & 148.1 & 48.5 & 99.6 \\
\hline $\mathrm{A} 2$ & 4062004.6 & 20556429.2 & 148.0 & 12.4 & 135.6 \\
\hline $\mathrm{A} 3$ & 4062047.7 & 20556401.2 & 147.2 & 58.2 & 89.0 \\
\hline $\mathrm{A} 4$ & 4062084.0 & 20556389.1 & 146.6 & 51.6 & 95.0 \\
\hline A5 & 4062097.7 & 20556442.4 & 148.0 & 34.6 & 113.4 \\
\hline
\end{tabular}

The testing scene is shown in Fig.6. Fig.7. shows the $x$ axis and $\mathrm{z}$ axis of the blasting vibration signals with an oscilloscope to observe the waveform picked up by the detector. After several tests, the effect is more ideal. The blasting source position can be located in 10 seconds after the detector picks up the $\mathrm{P}$ wave. One of the monitoring results is shown in Fig.8. The actual blasting position is at (20556904.3, 4061907.9, -129.4), and the system is located at $(20556898.6,4061903.2,-116.8)$, with a 17.99 meter spatial distance error.

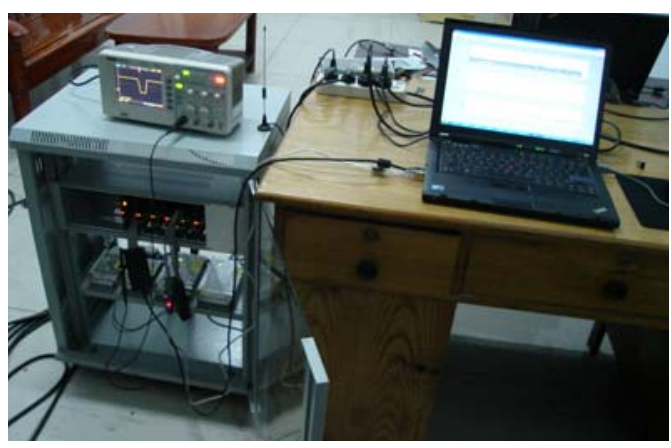

Fig.6. The testing scene

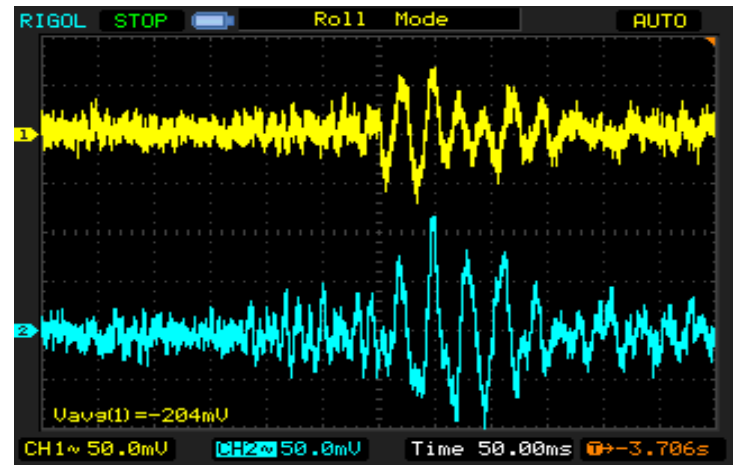

Fig.7. The $\mathrm{x}$ axis and $\mathrm{z}$ axis of the blasting vibration signals

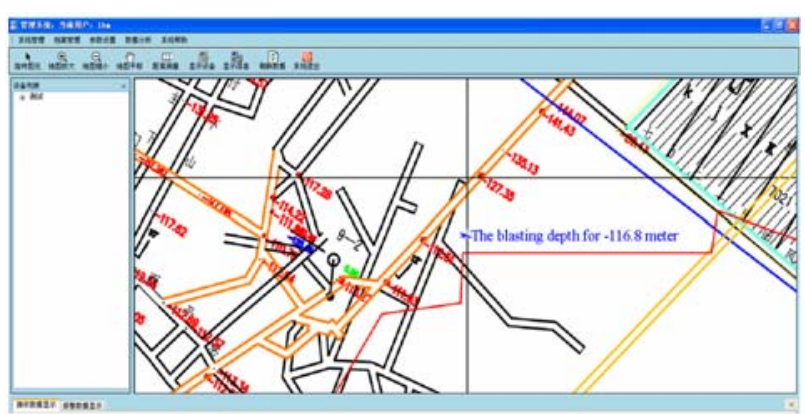

Fig.8. The test result in mine's electronic map

\section{CONCLUSION}

The method for monitoring the underground mining position by the blasting source location is first proposed. It is feasible by field proved method. The system can locate the explosion in 10 seconds after the sensor picks up the first $P$ wave, which can satisfy the demand of the in-time monitoring. The location errors are within 20 meters.

But the specific implementation scheme needs to be further improved. For example, the error of the detected wave-arriving time, the error between the actual average wave velocity and the average velocity used in the system, the error of the sensors' coordinates and so on should be taken into account. Besides, because the coefficient matrix of the linear position equations is decided by the reasonable distributions of the sensors, a more reasonable distribution can produce a better ill-condition, which leads to a smaller location error. 


\section{REFERENCES}

[1] Xin Qian (2008). Cross-border Mining: Curse of safety accidents. Safety and Healthy, 10, 16-17.

[2] Sun Yingjie (2008). The study of micro-seismic source place inversion methods. Published master dissertation, China University of Petroleum, Dongying, China.

[3] Zhang Naling (2010). Research on the image algorithms of the fracture of the micro-seismic monitoring oil well. Published master dissertation, Jilin University, Jinlin, China.

[4] Cheng Yunhai (2006). Preventing coal bump by applying micro-seismic Locating Monitoring Technology in long-wall face. Published doctoral dissertation, Shandong University of Science and Technology, Qingdao, China.

[5] Kania, M., Fereniec, M., Maniewski, R. (2007). Wavelet denoising for multi-lead high resolution ECG Signals. Measurement Science Review, 7 (4), 30-33.

[6] Naga Rajesh, A., Chandralingam, S., Anjaneyulu, T., Satyanarayana, K. (2012). Denoising EOG signal using stationary wavelet transform. Measurement Science Review, 12 (2), 46-51.

[7] Janusek, D., Kania, M., Zaczek, R., Zavala-Fernandez, H., Zbieć, A., Opolski, G., Maniewski, R. (2011). Denoising EOG signal using stationary wavelet transform. Measurement Science Review, 11 (6), 181184.
[8] Jena, D.P., Kumar, R. (2011). Implementation of wavelet de-noising and Image morphology on welding image for estimating HAZ and welding defects. Measurement Science Review, 11 (4), 108-111.

[9] Hussain, M.S., Mamun, Md. (2012). Effectiveness of the wavelet transform on the surface EMG to understand the muscle fatigue during walk. Measurement Science Review, 12 (1), 28-33.

[10] Phinyomark, A., Limsakul, C., Phukpattaranont, P. (2011). Application of wavelet analysis in EMG feature extraction for pattern classification. Measurement Science Review, 11 (2) 45-52.

[11] Cheng Zeng (2008). The research of image de-noising and enhancement based on wavelet theory. Published master dissertation, Wuhan University of Technology, Wuhan, China.

[12] Jinju Li, Zhifeng Ma, Qiongzhi Wu (2010). Image de noise based on the stationary wavelet translation with rotation. Laser \& Infrared, 40,1263-1268.

[13] Zheng Zhoushun, Huang Guanghui (2008). Conjugate vector base algorithm for solving ill-conditioned linear equations. Journal of Shandong University (Natural Science), 43, 1-5. 